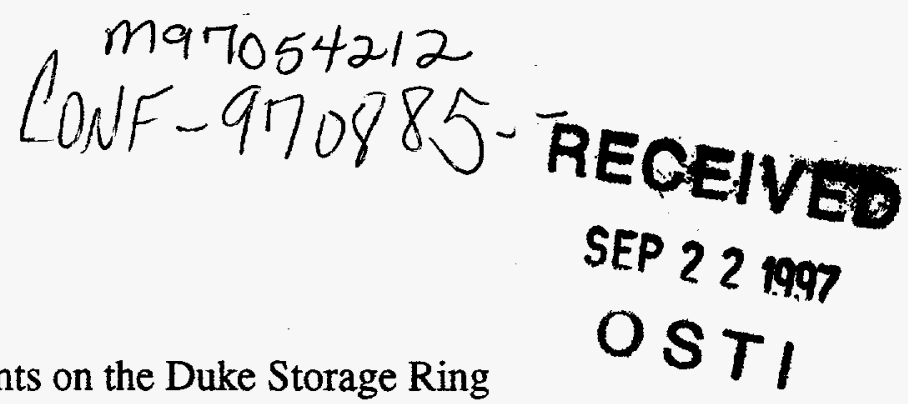

\title{
Initial Dual-Sweep Streak Camera Measurements on the Duke Storage Ring OK-4 UV/Visible FEL*
}

by

A. H. Lumpkin, B. X. Yang

Advanced Photon Source

Argonne National Laboratory

9700 S. Cass Ave. - Bldg. 401

Argonne, IL 60439 U.S.A.

Telephone: $630-252-4879$

FAX: $630-252-4732$

\author{
V. Litvinenko, B. Burnham, S. Park, P. Wang, and Y. Wu \\ Duke University FEL Laboratory \\ Durham, NC 27708 U.S.A.
}

Presented at FEL'97

Beijing, China

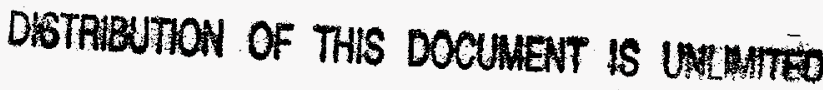

August 1997

*Work supported by the U.S. Department of Energy, Office of Basic Energy Sciences, under Contract No. W-31-109-ENG-38. 


\section{DISCLAIMER}

This report was prepared as an account of work sponsored by an agency of the United States Government. Neither the United States Government nor any agency thereof, nor any of their employees, makes any warranty, express or implied, or assumes any legal liability or responsibility for the accuracy, completeness, or usefulness of any information, apparatus, product, or process disclosed, or represents that its use would not infringe privately owned rights. Reference herein to any specific commercial product, process, or service by trade name, trademark, manufacturer, or otherwise does not necessarily constitute or imply its endorsement, recommendation, or favoring by the United States Government of any agency thereof. The views and opinions of authors expressed herein do not necessarily state or reflect those of the United States Government or any agency thereof. 


\section{DISCLAIMER}

Portions of this document may be illegible electronic image products. Images are produced from the best available original document. 


\title{
Initial Dual-Sweep Streak Camera Measurements on the Duke Storage Ring OK-4 UV/Visible FEL*
}

\author{
A. H. Lumpkin and B. X. Yang \\ Advanced Photon Source, Argonne National Laboratory \\ 9700 South Cass Avenue, Argonne, IL 60439 USA \\ V. Litvinenko, B. Burnham, S. Park, P. Wang, and Y. Wu \\ Duke University FEL Laboratory, Durham, NC 27708 USA
}

\begin{abstract}
Initial measurements at the Duke storage ring free electron laser (FEL) of both spontaneous emission radiation (SER) from the OK-4 and FEL radiation outcoupled from the oscillator cavity have been done with a dual-sweep streak camera. In the SER case, ANL's Hamamatsu C5680 streak camera was used to characterize stored electron-beam bunch lengths versus rf gap voltage and single-bunch current. Electron-beam bunch lengths were observed in a range from 50 to $300 \mathrm{ps}$ (FWHM). Particle beam energies of 270 and $500 \mathrm{MeV}$ in the storage ring (SR) were used with the OK-4 to generate SER from $500 \mathrm{~nm}$ to less than $200 \mathrm{~nm}$. Sensitivity to single-bunch, single-turn SER was shown down to $\sim 4 \mu \mathrm{A}$ beam current at $\lambda=450 \mathrm{~nm}$. By operating in the synchroscan mode and at bunch currents less than $1 \mathrm{~mA}$, beam orbit length vs. the resonator round-trip time was investigated by detecting the relative arrival time of the second pass outcoupled from the resonator at $\lambda \approx 200 \mathrm{~nm}$. These measurements were used in support of first lasing in the UV-visible regime in November 1996. The dual-sweep streak mode was then used to monitor lasing intensity variations on the $1-\mathrm{ms}$ and $50-\mathrm{ms}$ time scales, to measure a lasing pulse length as short as $\sim 3 \mathrm{ps}(\sigma)$ at $\lambda=388 \mathrm{~nm}$ at low beam current, and to track the orbit length detuning curve.
\end{abstract}

\footnotetext{
* Work supported by the U.S. Department of Energy, Office of Basic Energy Sciences, under Contract No. W-31 109-ENG-38.
} 


\section{INTRODUCTION}

In a collaborative effort between Duke University and the Advanced Photon Source (APS), the first dual-sweep streak camera images were taken of the output radiation of the Duke OK-4 FEL installed in the $1-\mathrm{GeV}$ storage ring [1]. These data were used to address directly the issues of the observed bunch length as a function of single-bunch current and if cavity gap voltage. For the available rf fields, a reasonable operating condition for adequate FEL gain was identified. Additionally, the key aspect of ring orbit length versus the resonator round-trip time was actually done with $\lambda=195 \mathrm{~nm}$ radiation outcoupled from the resonator, a wavelength shorter than the existing 239-nm FEL lasing record. The usefulness of these key measurements was confirmed as first lasing experiments were successful a few weeks later, although at $350-400 \mathrm{~nm}$ [2]. The dual-streak mode was also useful in monitoring lasing intensity variations on the 1-ms and 50-ms time scales, to measure a lasing pulse length as short as $\sim 3 \mathrm{ps}(\sigma)$ at $\lambda=388 \mathrm{~nm}$ at low electron beam current, and to track the orbit length detuning curve. These imaging experiments in a sense are extensions of techniques demonstrated on a linac-driven visible FEL oscillator in the late 1980s [3].

\section{EXPERIMENTAL BACKGROUND}

The Duke storage ring and OK-4 have been described elsewhere [1,2] and key design parameters are listed in Table 1. Operating energies from 0.25 to $1.0 \mathrm{GeV}$ are possible. In these experiments, the linac injection energy was about $270 \mathrm{MeV}$, and beam was stored at this energy or ramped in energy to $500 \mathrm{MeV}$. A key practical detail involved the ring's rf frequency of 178.547 $\mathrm{MHz}$, which is a subharmonic of the linac's s-band at $2856 \mathrm{MHz}$. To use the synchroscan streak plug-in at $119.0 \mathrm{MHz}$, the 24th subharmonic of 2856 , a special circuit was built at Duke which was phase-locked to the $178.5 \mathrm{MHz}$ clock [4]. The OK-4 was also critical to performing the very 
low (sub-0.1 mA) current measurements due to using its 68 periods rather than a single bending magnet.

As shown schematically in Fig. 1, the spontaneous emission radiation (SER) was initially picked off by a retractable mirror positioned upstream of the cavity mirror. Mirrors and/or beam splitters were also used to bring the cavity's outcoupled radiation to the APS streak camera. A monochromator that is not pictured was used to verify the operational wavelengths generated by the OK-4. The Hamamatsu C5680 dual-sweep streak camera was generally run with the vertical sweep in synchroscan mode and the horizontal sweep time selected for $10 \mu \mathrm{s}, 50 \mu \mathrm{s}, 1 \mathrm{~ms}$, or 50 ms. With the revolution frequency in the ring of $2.78 \mathrm{MHz}$, single-bunch, turn-by-turn data could readily be attained.

It also should be noted that the nominal operating range of the S-20 photocathode streak tube with the UV-transmitting input optics is from $200 \mathrm{~nm}$ to $800 \mathrm{~nm}$ [5]. However, there is a rapid decrease (factor of 30) in transmission of UV light through this input glass faceplate as the wavelength changes from $\lambda=300 \mathrm{~nm}$ to $\lambda=200 \mathrm{~nm}$. There is also a decrease in the photocathode quantum efficiency. This practical effect in detector sensitivity was a key part of our tests and why we originally looked at SER in the $400-500 \mathrm{~nm}$ regime in dual-sweep and then used synchronous averaging at $\lambda=200 \mathrm{~nm}$. In the visible regime we also used an etalon with 200 ps effective spacing to calibrate the $119.0 \mathrm{MHz}$ synchroscan plugin unit with this mainframe. A correction factor of $5 \%$ was determined. 


\section{EXPERIMENTAL RESULTS}

\subsection{Electron-Beam Bunch Length}

Initial tests on beam imaging were done with the bending magnet source, but we quickly moved on to the much stronger OK-4 source. A $450 \times 40 \mathrm{~nm}$ bandpass filter rejected most of the much weaker bending magnet's white radiation. A representative example of a dual-sweep streak image at $\lambda=450 \mathrm{~nm}$, beam energy $E_{B}=500 \mathrm{MeV}$, rf gap voltage $V_{\text {rf }}=500 \mathrm{kV}$, and beam current $I_{B}=0.19 \mathrm{~mA}$ is shown in Fig. 2. The vertical axis is the fast time axis with about 800 ps of the 1000-ps range displayed, and the horizontal sweep covers $10 \mu \mathrm{s}$. Each vertical image comes from a single pass through the OK-4. The selected fifth image's temporal profile has a calculated duration of $60 \mathrm{ps}$ (FWHM) or $25 \mathrm{ps}(\sigma)$. For comparison purposes, a similar image but with a lower gap voltage $V_{r f}=150 \mathrm{kV}$ and higher current $I_{B}=1.62 \mathrm{~mA}$ gives a measured bunch length much longer at 162 ps (FWHM) as shown in Ref. 6.

Figure 3 summarizes a series of measurements of bunch length with a fixed if gap voltage $(500 \mathrm{kV})$ and from the very low current regime $(0.003 \mathrm{~mA})$ to a current regime 900 times higher $(2.8 \mathrm{~mA}$ ). Significant (factor of 3) bunch lengthening is observed. It is noted that the plotted values are based on the off-line analysis using a Gaussian fit to the profile shape. For currents greater than $0.1 \mathrm{~mA}$, the preliminary analysis using the Hamamatsu MAC-TA algorithm, which finds the peak intensity and then searches for the two half-maximum intensity points, gave very similar results. Generally, there are ten beam images per file and two files were saved per parameter set. The off-line analysis on the actual profile shape as compared to a Gaussian shape was particularly useful for the very low intensity images. Based on available rf power, the $500 \mathrm{kV}$ gap voltage with a few $\mathrm{mA}$ average current resulted in 5-10 A peak current. This latter value was calculated to have adequate gain for lasing experiments [2]. 


\subsection{Cavity Length/Orbit Length Synchronism}

All measurements in the preceding section used the retractable pick-off mirror upstream of the resonator cavity mirror. In order to evaluate the feasibility of detecting radiation near $200 \mathrm{~nm}$, we initially used the pick-off mirror for wavelengths down to $220 \mathrm{~nm}$ in dual-sweep mode. We then changed to synchroscan sweep only and also changed a relay lens to a 50-mm focal length to provide a smaller focus at the entrance slit of the streak camera. The OK-4 was tuned to about $\lambda=$ $195 \mathrm{~nm}$, and the $193 \mathrm{~nm} \times 10 \mathrm{~nm}$ band pass filter was put in the path to the streak camera. The radiation outcoupled from the cavity was then transported to the streak camera. At that point manual tweaking of the resonator cavity mirrors was done until the observed signal in the monochromator was reduced by $50 \%$ between the resonator mirrors' being tuned and detuned. The if frequency for the storage ring was then varied to change the beam orbit length relative to the fixed resonator cavity length. As the stored beam current decreased, the bunch length shortened to about $40 \mathrm{ps}$ (FWHM). We could then clearly display the relative arrival time of the second pass in the resonator compared to the first pass of the next e-beam bunch. Figure 4 shows an example where the second pass or lower image is $99.5 \mathrm{ps}$ displaced from synchronism. By tracking the second pass's positions, the cavity and e-beam orbit length were synchronized. This preferred length was then used in subsequent lasing experiments within the next few weeks [2].

At the next level of synchronism was the mapping of the detuning curve under lasing conditions. In this case, although much finer steps in the rf frequency (orbit length) were taken, dramatic changes in the lasing temporal characteristics were observed. As shown in Fig. 5, the dualsweep streak images in the 800 -ps (vertical/ $\mathrm{T}_{1}$ ) by 1 -ms (horizontal $/ \mathrm{T}_{2}$ ) time scales exhibit a distinct $\mathrm{T}_{1}-\mathrm{T}_{2}$ tilt in the lasing intensities for $v_{\mathrm{rf}}=178.552872 \mathrm{MHz}$ (a) and $178.553070 \mathrm{MHz}$ (c), which is not evident in the synchronous case $v_{\mathrm{rf}}=178.552994 \mathrm{MHz}$ (b). Basically, the optical 
beam is "walking through" the electron beam longitudinal distribution over many turns in the desynchronized case. This "tilt" direction inverts when one adjusts the orbit length to the other side of synchronism. The relative intensity of lasing increases as tracked by the area under the lasing profile, and the bunch length shortens to $20 \mathrm{ps}$ (FWHM) with changes of less than $30 \mathrm{~Hz}$ as synchronism is attained for a stored beam current of $2-3 \mathrm{~mA}$. These phenomena are further addressed in References 7-9. Hama et al. discuss various detuning conditions for FEL operations at $479 \mathrm{~nm}$ and frequencies of $\pm 10 \mathrm{~Hz}$ from their nominal if frequency.

When using the dual sweep with 50 and $100 \mathrm{~ms}$ slow-axis time spans, the lasing was observed to oscillate in intensity at several ms intervals, which exhibited repeatability from one slow sweep trigger to the next. By adding a $60 \mathrm{~Hz}$ voltage oscillation to a steering magnet, the lasing could be turned on at about $120 \mathrm{~Hz}$ repetition frequency. As illustrated in Fig. 6, the simultaneous observation of the spontaneous emission from one pass through the OK-4 and picked off by a partly inserted extraction mirror and the lasing radiation outcoupled through the cavity mirror and window is possible. The lasing bunch length is typically 20 to $40 \mathrm{ps}$ (FWHM) in the upper images while the e-beam bunch length is $\sim 100$ ps (FWHM) in the lower SER image. For a well-tuned cavity and for low e-beam currents, the lasing at $\lambda=388 \mathrm{~nm}$ is observed over an even narrower time, $\sigma_{\mathrm{t}} \sim 3 \mathrm{ps}$ as described elsewhere in these proceedings [10]. This lasing micropulse bunchlength value is one of the shortest reported to date for a storage ring FEL.

\section{SUMMARY}

In summary, both the dual-sweep and synchroscan options of the streak camera were used to provide key information for the operation of this SR FEL. The source strength of the OK-4 SER was exploited to measure the electron-beam bunch lengths at very low currents $(<10 \mu \mathrm{A})$ and to detect radiation in the deep UV at $\lambda=195 \mathrm{~nm}$. The temporal effects during lasing clearly showed 
the sensitivity to If, power supply, and/or cavity stability. These results extend the range over which the techniques have been demonstrated on a FEL facility and support the proposals to apply such techniques at even shorter wavelengths in the future.

\section{ACKNOWLEDGMENTS}

The authors acknowledge John Galayda (ANL/APS/ASD) and John Madey (Duke FEL Laboratory) for their support of this collaboration.

\section{REFERENCES}

[1] V. Litvinenko et al., Nucl. Instrum. Methods A375, p. 46 (1996).

[2] V. Litvinenko et al., "First UV/Visible Lasing with the OK-4/Duke Storage Ring FEL: Design and Initial Performance," Presented at SPIE's LASE ‘97, FEL Challenges Conference, February 13, 1997, San Jose, CA.

[3] A. H. Lumpkin, Nucl. Instrum. Methods A296, p. 134 (1990).

[4] P. Wang, Duke University, unpublished information, October 1996.

[5] W. Cieslik, Hamamatsu Photonics Corp., C5680 Camera Data Sheets, June 1996.

[6] A. H. Lumpkin et al., "Initial Application of a Dual-Sweep Streak Camera to the Duke Storage Ring OK-4 Source," Proceedings of the 1997 Particle Accelerator Conference, Vancouver, B. C., Canada, May 12-16, 1997.

[7] V. Litvinenko et al., "OK-4/Duke Storage Ring FEL Operation in the UV," these Proceedings.

[8] R. Roux et al., "The Super ACO FEL Dynamics Measured with a Streak Camera," Proceedings of the 1996 FEL Conference, Rome, Italy, NIM (in press). 
[9] H. Hama et al., "Observation of Micro-Macro Temporal Structure and Saturation Mechanism on the UVSOR Free Electron Laser," Nucl. Instrum. Methods A358, p. 365 (1995).

[10] V. Litvinenko et al., "Microtemporal Structure in the OK-4/Duke Storage Ring UV FEL: Theory and Experiment," these Proceedings. 
Table 1: Summary of Parameters of the Duke Storage Ring and OK-4 (see Ref. 1)

\begin{tabular}{lc}
\hline \multicolumn{1}{c}{ Parameter } & Design Value \\
\hline Storage Ring & $0.25-1.0$ \\
Operating energy (GeV) & 107.46 \\
Ring circumference (m) & 2.789 \\
Revolution freq. (MHz) & 178.547 \\
rf frequency (MHz) & 0.1 \\
Beam current, A & $80-130$ \\
Peak current, A & $18 \times 10^{-9}$ \\
Horizontal emittance (m-rad) & $1 \times 10^{-9}$ \\
Vertical emittance, m-rad & 33 \\
Bunch length ( $\sigma, \mathrm{ps})$ & \\
OK-4 Parameter & 3.40 \\
Undulator length (m) & 0.10 \\
Period (m) & $0.0-5.8$ \\
Peak magnetic field (kG) & 0.34 \\
Buncher length (m) & $0-12$ \\
Magnetic field (kG) &
\end{tabular}




\section{Figure Captions}

Fig. 1. Schematic of the resonator setup on the Duke OK-4 storage ring FEL. A pick-off mirror inserted just upstream of the cavity mirror allows the sampling of the SER for each pass of the electron beam.

Fig. 2. Dual-sweep streak camera image of SER from the OK-4 at $\lambda=450 \mathrm{~nm}$, a beam energy of $500 \mathrm{MeV}, \mathrm{V}_{\mathrm{rf}}=500 \mathrm{kV}$, and $\mathrm{I}_{\mathrm{B}}=0.19 \mathrm{~mA}$. The time axes are $800 \mathrm{ps}$ vertical and $10 \mu \mathrm{s}$ horizontal. In this case, the bunch length of the selected image is $60 \mathrm{ps}$ (FWHM).

Fig. 3. Plot of the variation of beam bunch length with single-bunch current at $V_{\mathrm{rf}}=500 \mathrm{kV}$ and $\mathrm{E}=500 \mathrm{MeV}$. The Ok-4's SER intensity enabled the acquisition of data at less than $10 \mu \mathrm{A}$ with the streak camera.

Fig. 4. Synchroscan streak image of the SER outcoupled from the resonator cavity at $\lambda \sim 195 \mathrm{~nm}$. The very low current of $0.04 \mathrm{~mA}$ resulted in a bunch length of $44 \mathrm{ps}$ (FWHM). The cavity length is different from the orbit length by 99.5 ps as shown by the vertical displacement of the two images.

Fig. 5. Dual-sweep streak images of the FEL output for $v_{\text {If }}=178.552872 \mathrm{MHz}(\mathrm{a}), v_{\mathrm{rf}}=$ 178.552994 $\mathrm{MHz}$ (b), and 178.553070 $\mathrm{MHz}$ (c). The first example is desynchronized by about $13 \mu \mathrm{m}$ in orbit length from case $b$, and the optical pulse walks through the electron beam pulse in $100-200 \mu \mathrm{s}$. The vertical time span is $400 \mathrm{ps}$, and the horizontal time span is $1 \mathrm{~ms}$ in each of the images.

Fig. 6. Dual-sweep streak images of both the FEL output modulated by a $60 \mathrm{~Hz}$ kicker and the SER redirected by a partially inserted extraction mirror. The vertical time span is $400 \mathrm{ps,}$ and the horizontal time span is $100 \mathrm{~ms}$. The lasing bunch length is noticeably shorter than the electron beams as measured by SER. 


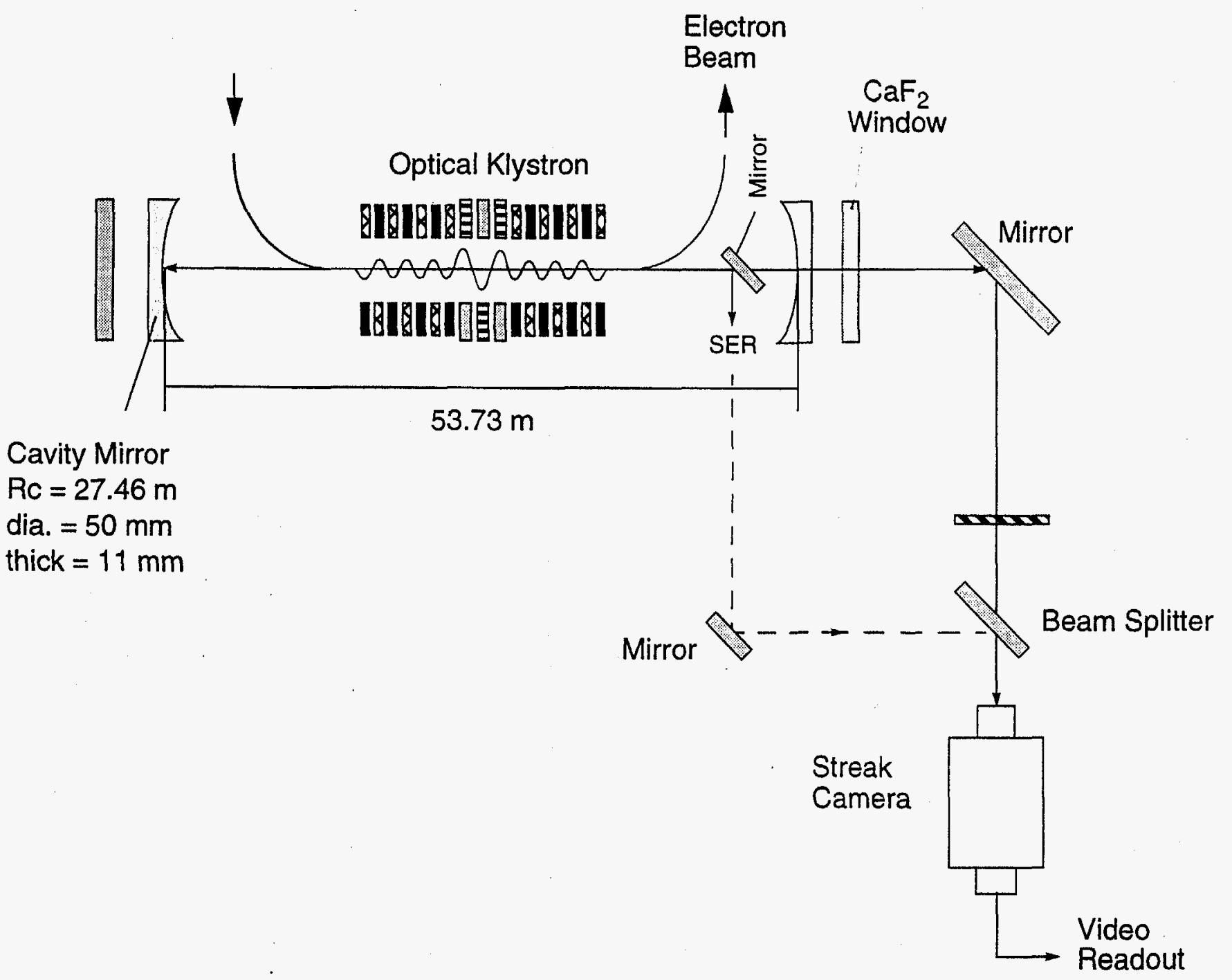

Fig. 1 


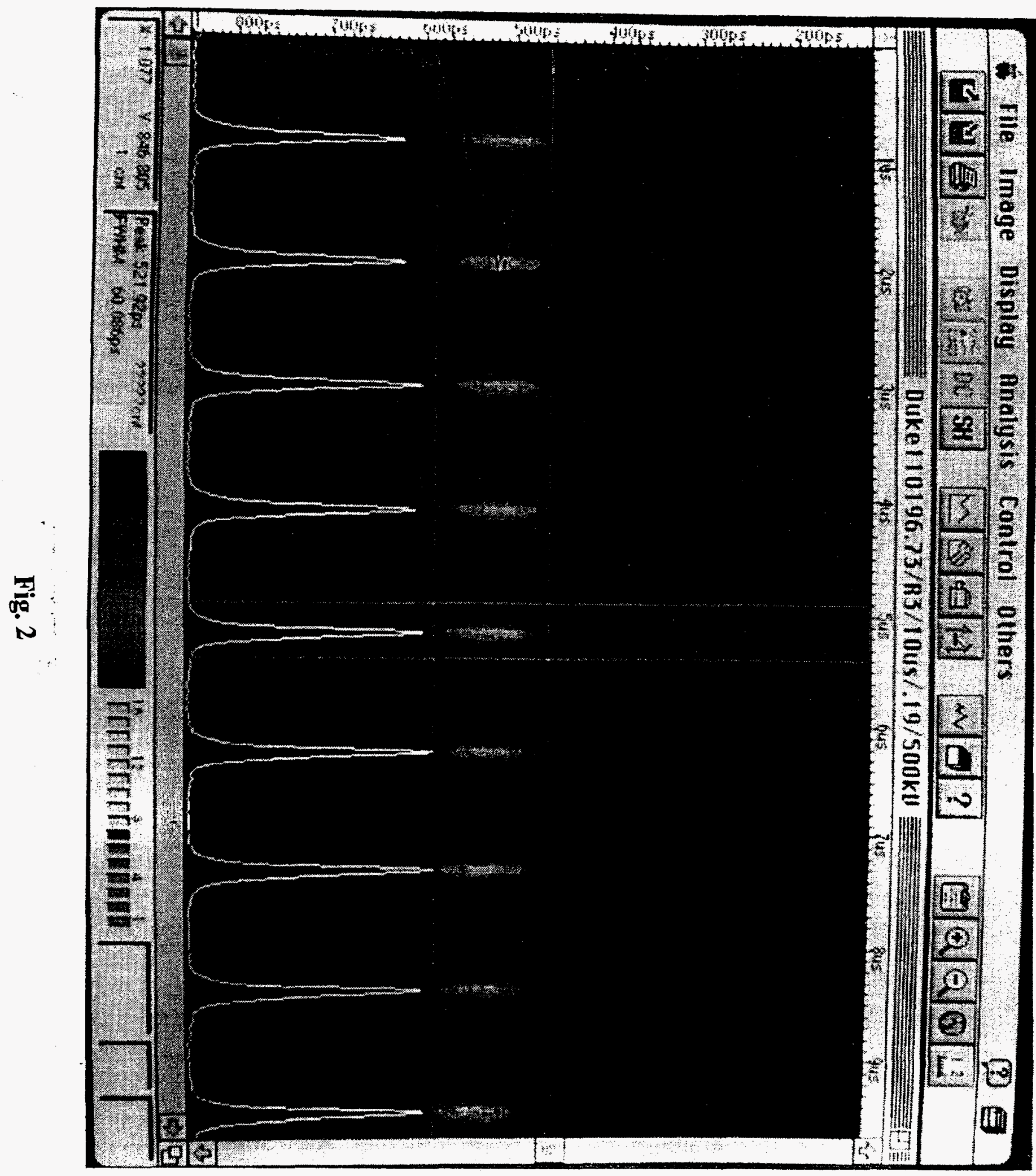




\section{Duke Storage Ring Bunch Length @ $500 \mathrm{MeV}$ (11/1/96, RF Gap Voltage 500 kV)}

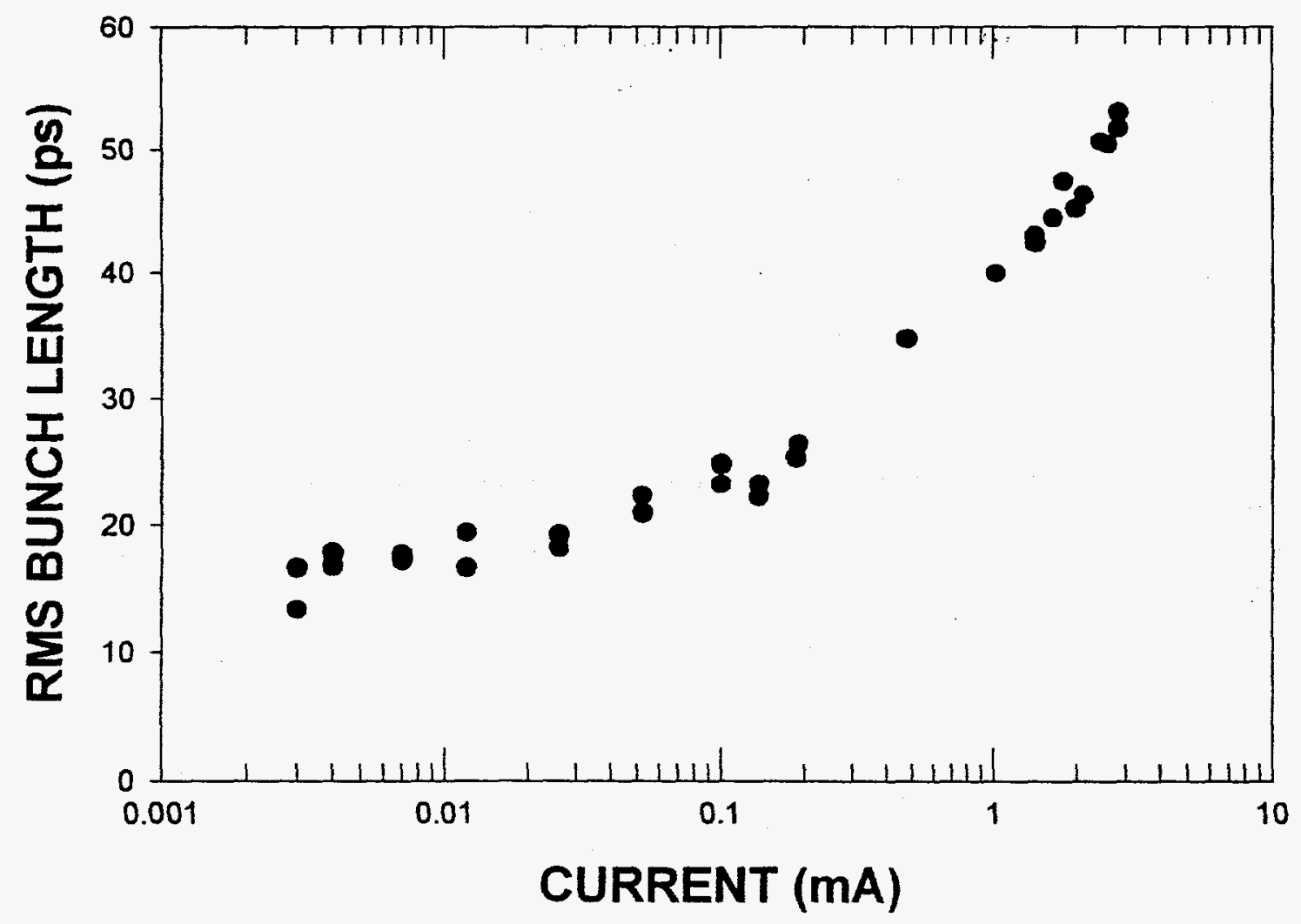

Fig. 3 


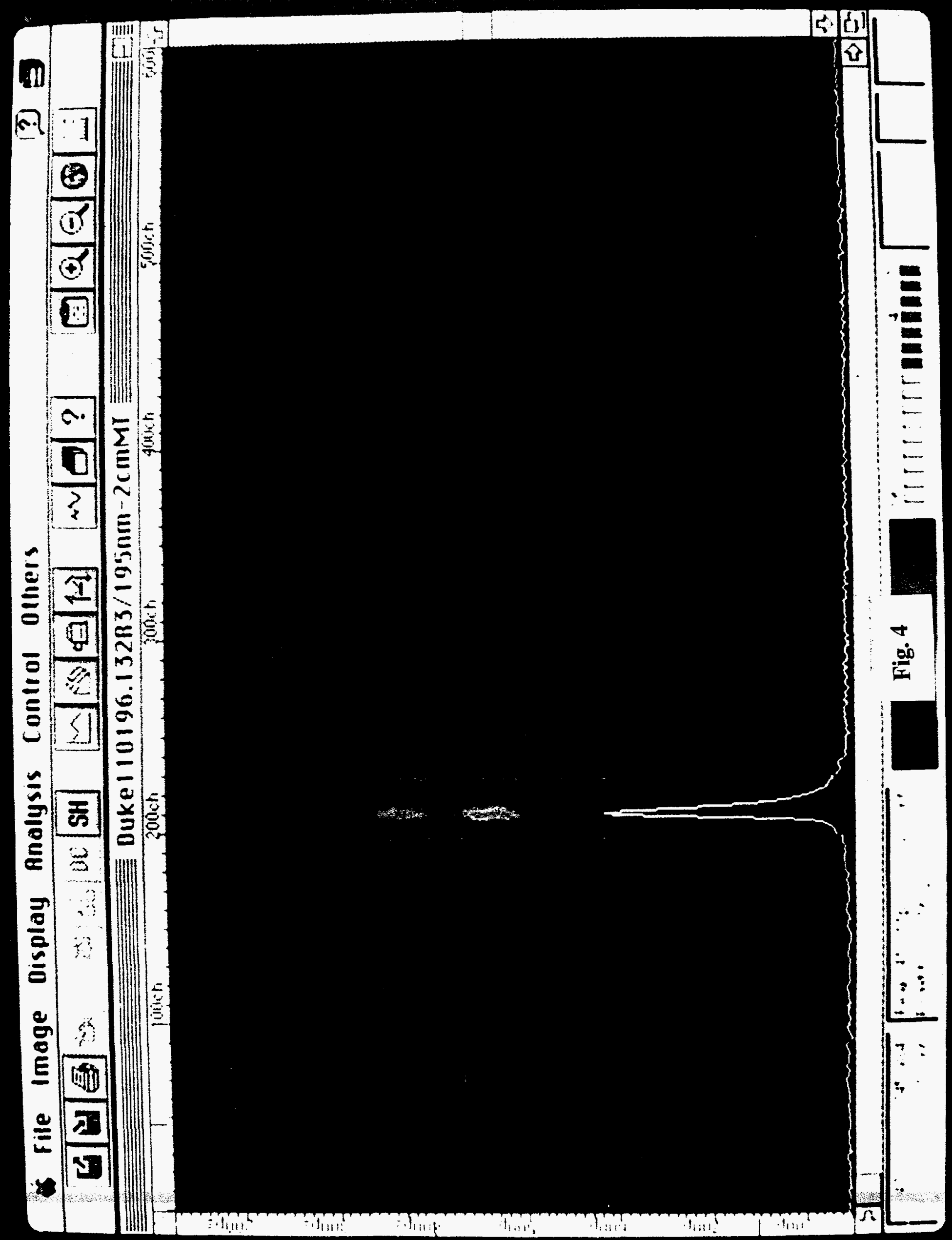



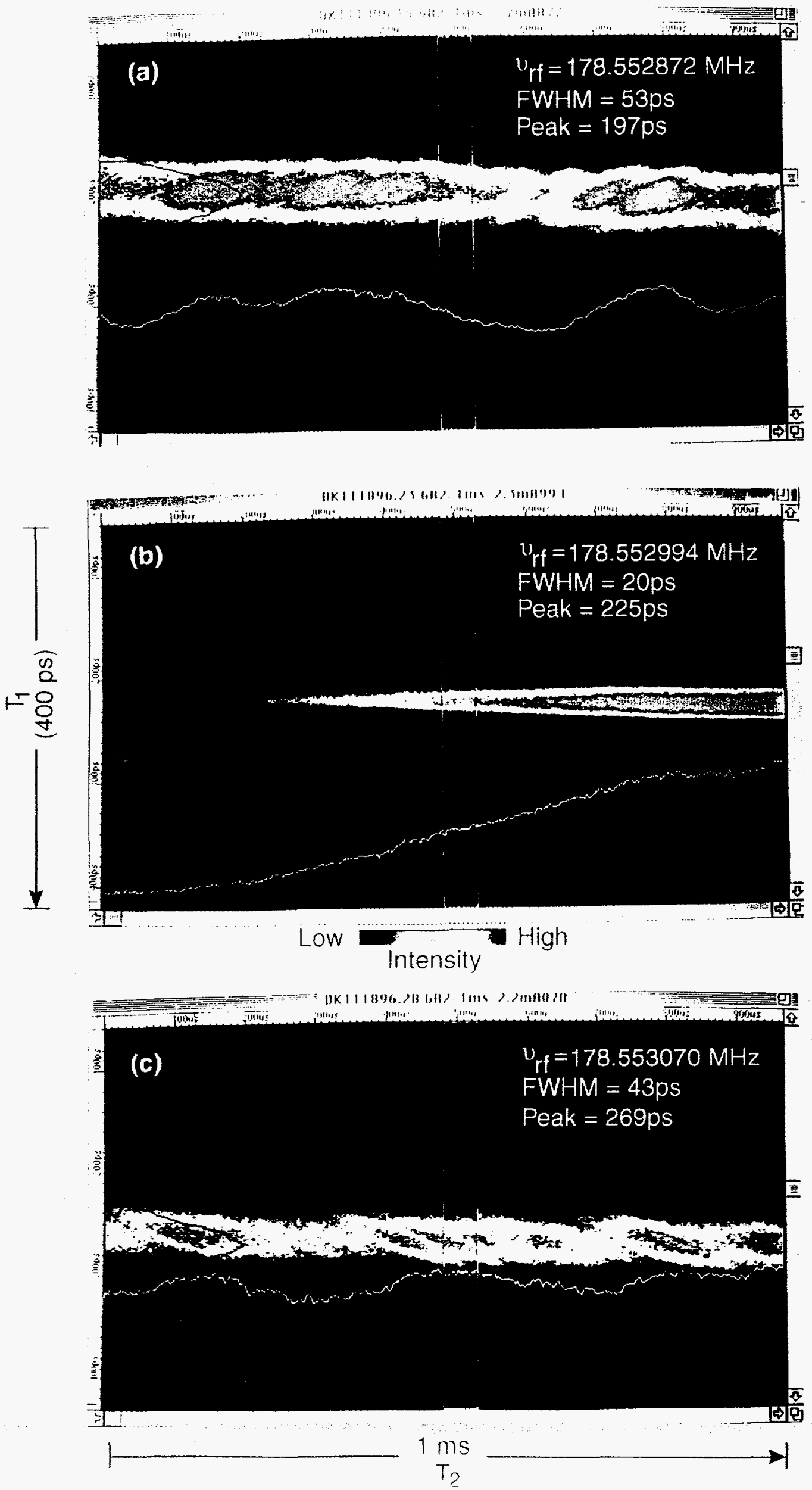

Fig. 5 


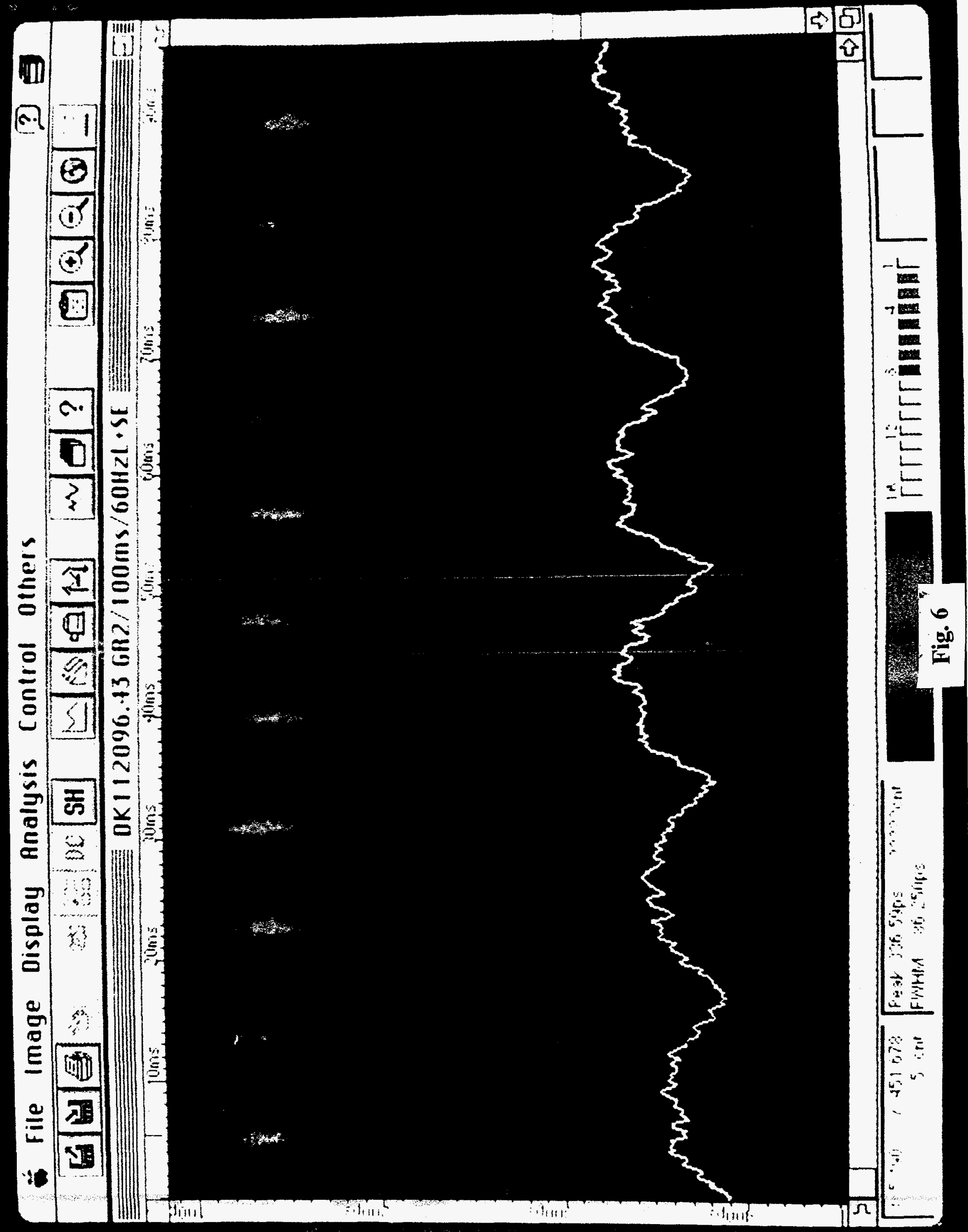

